\title{
Lie Superalgebraic Approach to Super Toda Lattice and Generalized Super KdV Equations*
}

\author{
Takeo Inami and Hiroaki Kanno ${ }^{\star \star}$ \\ Research Institute for Fundamental Physics, ${ }^{\star \star \star}$ Kyoto University, Kyoto 606, Japan
}

Received June 22, 1990; in revised form August 1, 1990

\begin{abstract}
We propose a super Lax type equation based on a certain class of Lie superalgebra as a supersymmetric extension of generalized (modified) $\mathrm{KdV}$ hierarchy. We are able to construct an infinite set of conservation laws and the consistent time evolution generators for generalized modified super $\mathrm{KdV}$ equations. The first few of the conserved currents, the (modified) super KdV equation and the super Miura transformation are worked out explicitly in the case of twisted affine Lie superalgebra $\operatorname{OSp}(2 \mid 2)^{(2)}$.
\end{abstract}

\section{Introduction}

Of integrable nonlinear systems generalized Korteweg-de Vries (KdV) equations and Toda lattice equations are particularly interesting classes in connection with conformal field theories. The Virasoro algebra can be extended to $W_{n}$ algebra [1] by incorporating conserved currents of higher spin. The $W_{n}$ algebra is known to arise from the Hamiltonian structure of the generalized $\mathrm{KdV}$ equation [2,3]. It has recently been shown [4] that perturbation of conformal field theories by certain types of interaction is described effectively by affine Toda lattice theories [5]. Some time ago Drinfeld and Sokolov developed a Lie algebraic method [2] to derive generalized $\mathrm{KdV}$ equations and relate them to affine Toda lattice equations. This method is also related to the method of coadjoint orbit and Hamiltonian reduction of current algebras.

In this paper we extend the Lie algebraic method of Drinfeld and Sokolov to the sypersymmetric case and develop a Lie superalgebraic method for generalized super KdV and super Toda lattice equations. Drinfeld and Sokolov introduced a

\footnotetext{
* Partially supported by the Grant-in-Aid for Scientific Research from the Ministry of Education, Science and Culture (\#01540246 and \#01790203). 
differential operator of first order

$$
\mathscr{L}=\partial+q(x)+\Lambda
$$

which takes values in an affine Lie algebra $\mathfrak{g}$. Here $q(x)$ is a field taking values in the Borel subalgebra $\mathfrak{b}$ of $\mathfrak{g}_{0}$ (0-th component in the standard gradation of $\mathfrak{g}$ ) and $\Lambda$ is the sum of generators in the simple root system (SRS) of $\mathfrak{g}$. They showed that the Lax type equation

$$
\partial_{t} \mathscr{L}=[\mathscr{A}, \mathscr{L}]
$$

gives a generalization of modified $\mathrm{KdV}(\mathrm{mKdV})$ hierarchy and constructed the time flow generator $\mathscr{A}$.

We will consider as a supersymmetric extension of the bosonic operator (1.1) the fermionic differential operator

$$
\mathscr{L}=D+q(x, \theta)+\Lambda
$$

which takes values in an affine Lie superalgebra $\mathfrak{g}$. Here $D=\partial / \partial \theta+\theta \partial / \partial x$ is the superderivative, $q(x, \theta)$ is a superfield taking values in the Borel subalgebra $\mathfrak{b}$ of $\mathfrak{g}_{0}$, and $\Lambda$ is the sum of generators of fermionic (Grassmann odd) SRS. Only special types of affine Lie superalgebra have a fermionic SRS of which the generators are all Grassmann odd (see Sect. 3) [6]. We propose the Lax type equations (1.2) and (1.3) as a generalization of the modified super $\mathrm{KdV}(\mathrm{msKdV})$ hierarchy. We are able to construct the conservation laws and the time evolution for generalized msKdV equations. Our construction relies on the assumption that $g$ admits a direct sum decomposition in terms of ad $\Lambda^{2}$ (see Eq. (5.3)). Presently we are not able to prove this assumption for an arbitrary affine Lie superalgebra possessing a fermionic SRS.

In Sect. 2 we introduce the basic notions of the Lie algebraic method for Toda lattice and generalized $\mathrm{KdV}$ equations. Those readers who are familiar with this method should skip this section. In Sect. 3, after recalling the basic notions of Lie superalgebras, we summarize super Toda lattice equations and propose generalized msKdV equations. In Sect. 4 we demonstrate that the super sine-Gordon equation, the super $\mathrm{KdV}$ equation and the super Miura transformation are obtained by considering the super Lax operator (1.3) associated with $O S p(2 \mid 2)^{(2)}$. We construct the conservation laws in Sect. 5 and the operator $\mathscr{A}$ used in the super Lax type equation (1.2) in Sect. 6.

\section{Lie Algebraic Approach to Generalized Modified KdV Equations}

In this section, we recapitulate the Lie algebraic method for the Toda lattice [7] and the generalized $\mathrm{KdV}$ equations [2]. For definiteness we consider the case of the affine Lie algebra $\mathfrak{g}=S L(r+1)^{(1)}$ associated with a simple Lie algebra $G=S L(r+1)$. Extension to other classes of affine Lie algebra requires slight modification of the method reviewed below.

The affine Lie algebra $\mathfrak{g}$ is the tensor product of the simple Lie algebra $G$ and the Laurent polynomial $C\left[\lambda, \lambda^{-1}\right]$. Let $\left\{E_{a}, F_{a}, H_{a}\right\}_{a=1}^{r}$ be the set of generators of $G$ in the Chevalley basis and $\left\{E_{a}, F_{a}, H_{a}\right\}_{a=0}^{r}$ that of $g$. Note that $E_{0}:=\lambda E_{\psi}\left(F_{0}:=\lambda^{-1} F_{\psi}\right)$, where $E_{\psi}\left(F_{\psi}\right)$ is the lowest (highest) root of $G$. The $g$ is 
equipped with $Z$ gradation [8]. The canonical gradation ${ }^{1}$

$$
\mathfrak{g}=\bigoplus_{k \in Z} \mathfrak{g}^{k}, \quad\left[\mathfrak{g}^{k}, \mathfrak{g}^{l}\right] \in \mathfrak{g}^{k+l}
$$

is defined by assigning degree $+1(-1)$ to $E_{a}\left(F_{a}\right)$. The standard gradation ${ }^{1}$ referring to the vertex $c_{0}$ of the Dynkin graph $\mathscr{D}$ of $\mathfrak{g}$,

$$
\mathfrak{g}=\bigoplus_{k \in Z} \mathfrak{g}_{k}, \quad\left[\mathfrak{g}_{k}, \mathfrak{g}_{l}\right] \in \mathfrak{g}_{k+l}
$$

is defined by assigning degree $+1(-1)$ to $E_{0}\left(F_{0}\right)$. The $\mathrm{g}_{0}$ is a semi-simple Lie algebra obtained by deleting the vertex $c_{0}$ from the $\mathscr{D} . \mathrm{g}_{0}=G$ in the present case of $\mathfrak{g}=S L(r+1)^{(1)}$. The Borel, Cartan and nilpotent subalgebras of $\mathrm{g}_{0}$ are defined by

$$
\begin{aligned}
& \mathfrak{b}=\mathfrak{g}_{0} \cap\left(\bigoplus_{k \leqq 0} \mathfrak{g}^{k}\right)=\mathfrak{h} \oplus \mathfrak{n}, \\
& \mathfrak{h}=\mathfrak{g}_{0} \cap \mathfrak{g}^{0}, \quad \mathfrak{n}=\mathfrak{g}_{0} \cap\left(\bigoplus_{k<0} \mathfrak{g}^{k}\right),
\end{aligned}
$$

respectively. The same definition as (2.3) will be used for Lie superalgebras in Sect. 3. A. Affine Toda Lattice. Let $\Phi_{a}(x, t)$ be an $(r+1)$-component field (of which only $r$ are independent) and set

$$
\Phi=\sum_{a=0}^{r} \Phi_{a} H_{a}
$$

Define

$$
\begin{aligned}
& \mathscr{L}=\partial+(\partial \Phi)+\Lambda, \\
& \overline{\mathscr{L}}=\partial_{t}+e^{-\Phi} \bar{\Lambda} e^{\Phi},
\end{aligned}
$$

where $\partial=\partial / \partial x$ and $\partial_{t}=\partial / \partial t$, and

$$
\Lambda=\sum_{a=0}^{r} E_{a}, \quad \bar{\Lambda}=\sum_{a=0}^{r} F_{a} .
$$

The zero curvature condition is written as

$$
0=[\mathscr{L}, \overline{\mathscr{L}}]=-\sum_{a=0}^{r}\left[\partial_{t} \partial \Phi_{a}-\exp \left(\sum_{b=0}^{r} K_{a b} \Phi_{b}\right)\right] H_{a},
$$

and it leads to the affine Toda lattice equation

$$
\partial_{t} \partial \Phi_{a}-\exp \left(\sum_{b=0}^{r} K_{b a} \Phi_{b}\right)=0,
$$

where $K_{a b}$ is the Cartan matrix of $\mathfrak{g}$.

B. Generalized Modified KdV Hierarchy. Consider the differential operator

$$
\mathscr{L}=\partial+q+\Lambda,
$$

1 The canonical and standard gradations are also called principal and homogeneous gradations, respectively 
where $q(x, t) \in C^{\infty}\left(\boldsymbol{R}^{2}, \mathfrak{b}\right)^{2} . \Lambda$ satisfies

$$
\Lambda^{r+1}=\lambda E,
$$

where $E$ is the identity.

An n-valued field $S$ defines a gauge transformation

$$
\mathscr{L} \rightarrow \tilde{\mathscr{L}}:=e^{\mathrm{ad} S}(\mathscr{L})=\partial+\tilde{q}+\Lambda, \quad \tilde{q} \in C^{\infty}\left(\boldsymbol{R}^{2}, \mathfrak{b}\right) .
$$

There exists a gauge transformation such that

$$
\mathscr{L} \rightarrow \mathscr{L}_{0}=e^{\operatorname{ad} U}(\mathscr{L})=\partial+q_{0}+\Lambda,
$$

where $q_{0}$ takes the form

$$
q_{0}=\sum_{i=0}^{\infty} f_{i} \Lambda^{-i} \in \operatorname{Ker} \operatorname{ad} \Lambda \equiv \mathscr{Z} .
$$

Here ad $\Lambda: \mathfrak{g}^{j} \rightarrow \mathrm{g}^{j+1}$ by $u \rightarrow[\Lambda, u]$.

Consider a time evolution equation

$$
\partial_{t} \mathscr{L}=[\mathscr{A}, \mathscr{L}]
$$

where $\mathscr{A}$ is to be determined by demanding consistent time evolution, i.e., that the right-hand side of Eq. (2.14) should belong to $\mathfrak{b}$. To construct the matrix $\mathscr{A}$ we define the centralizer of $\mathscr{L}, Z_{\mathscr{L}}=\left\{M \in C^{\infty}(\boldsymbol{R}, \hat{\mathrm{g}}) \mid[M, \mathscr{L}]=0\right\}$. Here $\hat{\mathrm{g}}=\mathrm{g}^{+} \oplus \mathrm{g}^{-}$, where $\mathfrak{g}^{+}=\bigoplus_{i \geqq 0} \mathfrak{g}^{i}$ and $\mathfrak{g}^{-}=\prod_{i<0} \mathfrak{g}^{i}$. By observing that for $M \in Z_{\mathscr{L}}$,

$$
\left[M^{+}, \mathscr{L}\right]=-\left[M^{-}, \mathscr{L}\right] \in C^{\infty}\left(\boldsymbol{R}^{2}, \mathfrak{b}\right)
$$

we see that Eq. (2.14) with $\mathscr{A}=M^{+}$is consistent. Noting $Z_{\mathscr{L}}=e^{- \text {ad } U}(\hat{\mathscr{Z}})$, we find that $\mathscr{A}$ is given by

$$
\mathscr{A}=\left(e^{-\operatorname{ad} U}(u)\right)^{+}, \quad u \in \hat{\mathscr{Z}} .
$$

In the present case of $\mathfrak{g}=S L(r+1)^{(1)}$ we have

$$
\mathscr{A}=\sum_{i=0}^{m} c_{i}\left(e^{-\operatorname{ad} U} \Lambda^{i}\right)^{+}, \quad c^{i} \in \boldsymbol{R} .
$$

Equation (2.14) leads to the same equation for the gauge equivalent class and it is called generalized $\mathrm{KdV}$ hierarchy associated with $\left(\mathfrak{g}, c_{0}\right)$. We consider two typical gauge fixed operators $\mathscr{L}^{\text {can }}$ and $\mathscr{L}^{\text {diag }}$ defined by $\mathscr{L}$ in which $q$ takes the form

$$
q^{\mathrm{can}}(x, t)=u_{1} e_{1, r+1}+u_{2} e_{2, r+1}+\cdots+u_{r} e_{r, r+1}
$$

and

$$
q^{\mathrm{diag}}(x, t)=\sum_{a=1}^{r} v_{a} H_{a}=\operatorname{diag}\left(q_{1}, \ldots, q_{r+1}\right) \in \mathfrak{h}
$$

respectively. Here we have taken the $(r+1) \times(r+1)$ matrix representation in which

${ }^{2}$ We sometimes write $C^{\infty}(R, \mathfrak{b})$ instead of $C^{\infty}\left(R^{2}, \mathfrak{b}\right)$ when we are only interested in the $x$ dependence 
the Chevalley generators are given by $E_{a}=e_{a+1, a}, F_{a}=e_{a, a+1}(a \neq 0)$, where $e_{a, b}$ denotes the matrix having unity at the $(a, b)$ site and zeros elsewhere. $q^{\text {can }}$ satisfies the equation

$$
\partial q^{\mathrm{can}} / \partial t=F\left(q^{\mathrm{can}}, \partial q^{\mathrm{can}} / \partial x, \ldots\right)
$$

where $F$ is a differential polynomial in $q^{\text {can }}$. Equation $(2.20)$ is a coordinate realization of the generalized KdV hierarchy. Expand $e^{-\operatorname{ad} U}(u)$ as

$$
e^{-\mathrm{ad} U}(u)=\sum_{i=-\infty}^{m} A^{i}, \quad A^{i} \in \mathrm{g}^{i}
$$

$q^{\text {diag }}$ satisfies the equation

$$
\partial q^{\text {diag }} / \partial t=-\partial A^{0}
$$

where $A^{0}$ is a differential polynomial in $q^{\text {diag }}$. Equation (2.22) is called generalized modified $\mathrm{KdV}$ equation. The map relating to each operator $\mathscr{L}^{\text {diag }}$ its gauge equivalent class $\mathscr{L}^{\text {can }}$ is called generalized Miura transformation.

C. Scalar Lax Equation and Generalized Miura Transformation. We introduce a $B\left(\left(\partial^{-1}\right)\right)$-module structure on $B\left(\left(\lambda^{-1}\right)\right)^{r+1}$ as follows. We use the notation $B:=C^{\infty}(R, C)$ (ignoring the $t$-independence) and

$$
\begin{aligned}
B[\lambda]: & =\left\{\sum_{i=0}^{n} a_{i} \lambda^{i} \mid a_{i} \in B, n \geqq 0\right\}, \\
B\left(\left(\lambda^{-1}\right)\right): & =\left\{\sum_{i=-\infty}^{n} a_{i} \lambda^{i} \mid a_{i} \in B, n \in Z\right\} .
\end{aligned}
$$

Elements of $B\left(\left(\partial^{-1}\right)\right)$ are called pseudodifferential symbols. For $\eta \in B\left(\left(\lambda^{-1}\right)\right)^{r+1}$ and

$$
P=\sum_{i=0}^{n} b_{i} \partial^{i} \in B[\partial]
$$

we define the action of $P$ on $\eta$ by

$$
P \cdot \eta:=\sum_{i=0}^{n} b_{i} \mathscr{L}^{i}(\eta)
$$

Let $\psi_{1}=(1,0, \ldots, 0)^{t} \in B[\lambda]^{r+1}$. We can show that each element of $B[\lambda]^{r+1}$ can be uniquely represented in the form $P \cdot \psi_{1}$. To each $\mathrm{g}$-valued Lax operator $\mathscr{L}$ of the form (2.9) we assign a scalar Lax operator $L$ of the form

$$
L=\partial^{r}+\sum_{i=0}^{r-1} U_{i} \partial^{i}
$$

by setting

$$
\lambda \psi_{1}=L \cdot \psi_{1}=\left(\mathscr{L}^{r}+\sum_{i=0}^{r-1} U_{i} \mathscr{L}^{i}\right) \psi_{1} .
$$

Note that to the gauge equivalent set of operators $\mathscr{L}$ corresponds the same scalar Lax operator $L$.

The generalized Miura transformation can be derived by referring to the matrix 
representation in which we have

$$
\Lambda=\left(\begin{array}{cccccc}
0 & 0 & 0 & \cdots & 0 & \lambda \\
1 & 0 & 0 & \cdots & 0 & 0 \\
0 & 1 & 0 & \ddots & 0 & 0 \\
\vdots & \ddots & \ddots & \ddots & \ddots & \vdots \\
0 & 0 & \cdots & 1 & 0 & 0 \\
0 & 0 & \cdots & 0 & 1 & 0
\end{array}\right)
$$

Take the standard basis $\left\{\psi_{1}, \ldots, \psi_{r+1}\right\}$ in $B[\lambda]^{r+1}$. In the canonical reduction (2.18), noting

$$
\Lambda^{i} \psi_{1}=\psi_{1+i} \quad(i<r)
$$

we find that $U_{i}=-u_{i}$. In the diagonal reduction (2.19) we have $\left(\mathscr{L}-q_{i}\right) \psi_{i}=\psi_{i+1}$ and hence

$$
\left(\mathscr{L}-q_{r}\right) \cdots\left(\mathscr{L}-q_{2}\right)\left(\mathscr{L}-q_{1}\right) \psi_{1}=\lambda \psi_{1},
$$

which implies the generalized Miura transformation

$$
L=\left(\partial-q_{r}\right) \cdots\left(\partial-q_{2}\right)\left(\partial-q_{1}\right) .
$$

The equation (2.14) for the gauge equivalence class coincides with the scalar Lax equation

$$
\partial_{t} L=[A, L]
$$

where $A$ is given by

$$
A=-\sum_{i=0}^{m} c_{i}\left(L^{i / r}\right)^{+} .
$$

D. Conservation Laws. Write Eq. (2.14) in the form $\left[\partial_{t}-\mathscr{A}, \mathscr{L}\right]=0$. Then

$$
\left[\partial_{t}-\tilde{\mathscr{A}}, \mathscr{L}_{0}\right]=0
$$

where $\tilde{\mathscr{A}}=e^{\text {ad } U}(\mathscr{A}-\partial / \partial t)+\partial / \partial t$. This equation is written in the form

$$
\partial q_{0} / \partial t+\partial \tilde{\mathscr{A}}=0
$$

Equation (2.35) implies that the coefficients $f_{i}$ in Eq. (2.13) are densities of conservation laws for Eq. (2.14).

\section{Super Toda Lattice and Generalized Super KdV Equations}

Toda lattice equations with spacetime supersymmetry were constructed by several authors [6,9] employing the notion of super principal embedding $O S p(1 \mid 2) \subset G$, where $G$ is a Lie superalgebra. Manin and Radul suggested that a generalization of super $\mathrm{KdV}$ equation can be obtained as a reduction of a super KP hierarchy [10]. Lie algebraic aspects of generalized super $\mathrm{KdV}$ equations are unclear in their derivation using scalar super Lax operators. We will propose a Lie superalgebraic method for generalized (modified) super $\mathrm{KdV}$ equations in which they are related to super Toda lattice equations. 
We first give a brief summary of the Lie superalgebraic method for super Toda lattice equations introducing basic notions of Lie superalgebras [11].

Let $G$ be a finite dimensional Lie superalgebra of rank $r$ and

$$
\left\{E_{a}, F_{a}, H_{a}\right\}_{a=1}^{r}
$$

the generators in its Chevalley basis. We consider the super principal embedding $\operatorname{OSp}(1 \mid 2) \subset G$, where the Chevalley generators $\{J, \bar{J}, H\}$ of $O S p(1 \mid 2)$ are given by

$$
\begin{aligned}
H & =\sum_{a=1}^{r} c_{a} H_{a}, \\
J & =\sum_{a=1}^{r} d_{a} E_{a}, \quad \bar{J}=\sum_{a=1}^{r} \bar{d}_{a} F_{a},
\end{aligned}
$$

with

$$
c_{a}=d_{a} \bar{d}_{a}=\sum_{b=1}^{r}\left(K^{-1}\right)_{b a} .
$$

The super principal embedding $O S p(1 \mid 2) \subset G$ exists only when one can choose a purely fermionic (Grassmann odd) simple root system (SRS) for $G$. This is possible only for limited classes of Lie superalgebra. To each super principal embedding specified by the Cartan matrix $K_{a b}$ corresponds a non-affine Toda lattice with spacetime supersymmetry (super Toda lattice in short) $[6,9]$.

The argument given above can be extended to the case of infinite-dimensional Lie superalgebra (affine Lie superalgebra). An affine super Toda lattice corresponds to an affine Lie superalgebra having an SRS which leads to a sum of Lie superalgebras admitting super-principal embedding after deleting any of the vertices of its Dynkin graph.

The simple and affine Lie superalgebras which possess a purely fermionic SRS have been listed by Leites, Saveliev and Serganova [6]:

Finite-dimensional:

$$
S L(n \pm 1 \mid n) ; \quad O S p(m \mid 2 n) \quad(m=2 n, 2 n+2,2 n \pm 1) ; \quad D(2 \mid 1 ; \alpha)
$$

Infinite-dimensional:

$$
\begin{aligned}
& S L(n \mid n)^{(1)} ; \quad O S p(2 n+2 \mid 2 n)^{(1)} ; \quad D(2 \mid 1 ; \alpha)^{(1)} \\
& S Q(2 n+1)^{(2)} ; \quad S L(n \mid n)^{(2)} ; \quad O S p(2 n \mid 2 n)^{(2)}
\end{aligned}
$$

In general several inequivalent SRS's correspond to a Lie superalgebra ${ }^{3}$, as illustrated in Fig. 1. An affine super Toda lattice equation is obtained by using the purely fermionic SRS of one of the affine Lie superalgebras listed in (3.5). We use the same fermionic SRS's to construct generalized modified super $\mathrm{KdV}$ equations associated with an affine Lie superalgebra.

Let $g$ be one of the affine Lie superalgebras listed in (3.5). We take the Chevalley generators of its purely fermionic SRS

$$
\left\{E_{a}, F_{a}, H_{a}\right\}_{a=0}^{r}
$$

${ }^{3}$ A fairly complete list of Dynkin graphs of simple and affine Lie superalgebras is given in refs. [6] and [12] 


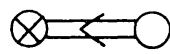

(a)

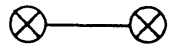

(b)

Fig. 1. Two inequivalent Dynkin graphs of the simple Lie superalgebra $\operatorname{OSp}(2 \mid 2)$. We have followed the convention of ref. [6]

We introduce $Z$ gradation in $g$ in the same way as the bosonic case (2.1) and (2.2). We assign degree $+1(-1)$ to $E_{m}\left(F_{m}\right)$ in the standard gradation (2.2) referring to the vertex $c_{m}$. Note that in the canonical gradation (2.1), elements of even (odd) degree are Grassmann even (odd). The Borel, Cartan and nilpotent subalgebras $\mathfrak{b}, \mathfrak{h}$ and $\mathfrak{n}$ are defined in the same way as the bosonic case, Eq. (2.3).

Let $\Phi_{a}\left(x, \theta ; t, \theta_{t}\right)$ be an $(r+1)$-component scalar superfield (of which only $r$ are independent), and set

$$
\Phi=\sum_{a=0}^{r} \Phi_{a} H_{a} \in \mathfrak{h} .
$$

Consider a pair of super-differential operators

$$
\begin{aligned}
& \mathscr{L}=D+(D \Phi)+\Lambda, \\
& \overline{\mathscr{L}}=D_{t}+e^{-\Phi} \bar{\Lambda} e^{\Phi},
\end{aligned}
$$

where

$$
D:=\partial / \partial \theta+\theta \partial / \partial x, \quad D_{t}:=\partial / \partial \theta_{t}+\theta_{t} \partial / \partial t,
$$

and

$$
\Lambda:=\sum_{a=0}^{r} E_{a}, \quad \bar{\Lambda}:=\sum_{a=0}^{r} F_{a} .
$$

The operators $\mathscr{L}$ and $\overline{\mathscr{L}}$ are Grassmann odd and hence $\Lambda$ and $\bar{\Lambda}$ must also be so. This means that the SRS (3.6) must be purely fermionic, as remarked above.

Given the pair of super Lax operators (3.8), we now write down the zero-curvature condition in the Zakharov-Shabat scheme [13]

$$
[\mathscr{L}, \overline{\mathscr{L}}]_{+}=0 .
$$

This condition yields super Toda lattice equations,

$$
D_{t} D \Phi_{a}=-\exp \left(\sum_{b=0}^{r} K_{b a} \Phi_{b}\right) .
$$

This equation is written in terms of superfields and superderivatives and hence it is manifestly supersymmetric.

We now turn to the construction of generalized modified super $\mathrm{KdV}$ (msKdV) equations. This can be made by extending Drinfeld and Sokolov's method to the case of affine Lie superalgebras $\mathfrak{g}$.

We consider as a supersymmetric extension of the differential operator (2.9) the super-differential operator

$$
\mathscr{L}=D+q(x, \theta ; t)+\Lambda .
$$


Here $D$ is defined in Eq. (3.9). $q(x, \theta ; t)$ is a fermionic superfield taking values in the Borel subalgebra $\mathfrak{b}$ of $\mathfrak{g}_{0} . \Lambda$ is given precisely by Eq. (3.10). In order that the super Lax operator (3.13) makes sense $\Lambda$ has to be Grassmann odd, and hence all $E_{a}$ must be so. This is possible only when $\mathrm{g}$ is one of those affine Lie superalgebras having a purely fermionic SRS considered above. Recall that the Lax operator $\mathscr{L}$ of the bosonic $\mathrm{mKdV}$ equation and that of the bosonic affine Toda lattice are related by diagonal reduction [2]. The same relation holds for the super Lax operator (3.13) and that of the affine super Toda lattice.

We propose that the super Lax type equation

$$
\partial_{t} \mathscr{L}=[\mathscr{A}, \mathscr{L}]
$$

gives a generalization of the msKdV equation. We have to show that Eq. (3.14) is a sensible nonlinear equation having the same desirable properties as generalized $\mathrm{mKdV}$ equations. To this end we will construct in Sects. 5 and 6 the conservation laws and the time flow generator $\mathscr{A}$ which gives a consistent time evolution.

\section{4. $\operatorname{OSp}(2 \mid 2)^{(2)}$ and Super Sine-Gordon and Super (Modified) KdV Equations}

Before proceeding to the formal arguments in Sects. 5 and 6, we consider in this section the simplest case of $\mathfrak{g}=\operatorname{OSp}(2 \mid 2)^{(2)}$ and demonstrate that the super sine-Gordon $[14,15]$ and super $\mathrm{KdV}$ equations $[10,16]^{4}$ derived previously in non-Lie algebraic methods are obtainable by considering the super Lax operators (3.8) and (3.13).

The simple Lie superalgebra $G=\operatorname{OSp}(2 \mid 2)$, Lie algebra of $N=2$ super Möbius transformations, is $(4,4)$-dimensional and has rank 2 . It has two inequivalent SRS's (the associated Dynkin graphs are shown in Fig. 1). We take the purely fermionic SRS (Fig. 1b) and denote its Chevalley generators by $\left\{e_{a}, f_{a}, h_{a}\right\}_{a=1}^{2}$. We will use the Cartan matrix of the form

$$
K_{a b}=\left(\begin{array}{ll}
0 & 2 \\
2 & 0
\end{array}\right) .
$$

The commutation relations of the generators are given in Appendix A.

The twisted affine Lie superalgebra $\mathfrak{g}=\operatorname{OSp}(2 \mid 2)^{(2)}$ is defined referring to the $Z_{2}$ symmetry of the Dynkin graph $1 \mathrm{~b}$ of $G$, as explained in Appendix A. The Chevalley generators are

$$
\begin{aligned}
& E_{1}=e_{1}+e_{2}, \quad E_{0}=\lambda\left(f_{1}-f_{2}\right), \\
& F_{1}=f_{1}+f_{2}, \quad F_{0}=\lambda^{-1}\left(e_{1}-e_{2}\right), \\
& H_{1}=H_{0}=h_{1}+h_{2}=H .
\end{aligned}
$$

The Cartan matrix is

$$
K_{a b}=\left(\begin{array}{cc}
2 & -2 \\
-2 & 2
\end{array}\right) \text {. }
$$

${ }^{4}$ Two types of "super" KdV equation are known. One has supersymmetry in space [16] and the other does not [17]. We are concerned with the first one 
We introduce a single scalar superfield $\Phi$ and define

$$
\Lambda:=E_{0}+E_{1}, \quad \bar{\Lambda}:=F_{0}+F_{1} .
$$

The super differential operators are

$$
\begin{aligned}
\mathscr{L} & =D+(D \Phi) H+\Lambda, \\
\overline{\mathscr{L}} & =D_{t}+\exp (-\Phi H) \bar{\Lambda} \exp (\Phi H) \\
& =D_{t}+\exp 2 \Phi F_{1}+\exp (-2 \Phi) F_{0} .
\end{aligned}
$$

The zero curvature condition (3.11) gives the super sine-Gordon equation

$$
D D_{t} \Phi=2 \cosh (2 \Phi)
$$

We now turn to the derivation of the modified super $\mathrm{KdV}$ equation. This will be made by relating the super Lax operator $\mathscr{L}$ to a scalar super Lax operator $L$. To this end we will introduce a super $\mathscr{D}$-module structure by using an explicit matrix representation of $\operatorname{OSp}(2 \mid 2)^{(2)}$ (see Subsect. 2.C).

The Borel subalgebra $\mathfrak{b}=\mathfrak{h} \oplus \mathfrak{n}$ is 3 -dimensional, $\mathfrak{h}$ being spanned by $H$ and $\mathfrak{n}$ by $F_{1}$ and $F_{0}$. In the same fashion as the bosonic case (2.11), an $n$-valued superfield $S$ defines gauge transformation. The $n$ is two dimensional and we have only one dynamical degree of freedom. In parallel with the bosonic case we consider two typical gauge fixed operators $\mathscr{L}^{\text {can }}$ and $\mathscr{L}^{\text {diag }}$ defined by (3.13) with $q$ of the form

$$
\begin{aligned}
q^{\mathrm{can}} & =W(x, \theta, t) \cdot F_{1}{ }^{2}, \\
q^{\mathrm{diag}} & =\Psi(x, \theta, t) \cdot H,
\end{aligned}
$$

respectively, and identify the super integrable non-linear equations corresponding to these gauge fixed operators. This can be made by taking the $4 \times 4$ matrix realization of the Chevalley generators given in Appendix A. We have

$$
\begin{aligned}
\Lambda & =\left(\begin{array}{cccc}
0 & 0 & \lambda & 1 \\
0 & 0 & -\lambda & 1 \\
1 & 1 & 0 & 0 \\
\lambda & -\lambda & 0 & 0
\end{array}\right), \\
H & =\left(\begin{array}{cccc}
0 & 0 & 0 & 0 \\
0 & 0 & 0 & 0 \\
0 & 0 & 2 & 0 \\
0 & 0 & 0 & -2
\end{array}\right), \quad F_{1}{ }^{2}=\left(\begin{array}{cccc}
0 & 0 & 0 & 0 \\
0 & 0 & 0 & 0 \\
0 & 0 & 0 & 0 \\
0 & 0 & -2 & 0
\end{array}\right) .
\end{aligned}
$$

Accordingly the super Lax operators $\mathscr{L}^{\text {can }}$ and $\mathscr{L}^{\text {diag }}$ are also $4 \times 4$ matrices. We note that

$$
\Lambda^{4}=4 \lambda^{2} E
$$

where $E$ is the $4 \times 4$ unit matrix.

Using the $4 \times 4$ matrix realization, we introduce a super $\mathscr{D}$-module structure on $\mathscr{B}\left(\left(\lambda^{-1}\right)\right)^{4}$, where $\mathscr{B}$ is the algebra of superfields, as follows. An element $\psi$ of $\mathscr{B}\left(\left(\lambda^{-1}\right)\right)^{4}$ is four-component column vector $\psi=\left(\psi_{1}, \ldots, \psi_{4}\right)^{t}$, where each component is a superfield with a $\lambda$ expansion of the form

$$
\psi_{i}=\sum_{k=-n_{i}}^{\infty} \psi_{i}^{(k)}(x, \theta) \lambda^{-k} .
$$


The super Lax operator $\mathscr{L}$ acts on $\mathscr{B}\left(\left(\lambda^{-1}\right)\right)^{4}$. For a super differential operator of the form $P=D^{n}+U_{n-1} D^{n-1}+\cdots+U_{0}$, we define an action of $P$ on $\mathscr{B}\left(\left(\lambda^{-1}\right)\right)^{4}$ by

$$
P \cdot \psi=\sum_{k=0}^{n} U_{k} \mathscr{L}^{k} \psi, \quad\left(\psi \in \mathscr{B}\left(\left(\lambda^{-1}\right)\right)^{4}\right) .
$$

It is easy to see that this action allows us to define a super $\mathscr{D}$-module structure on $\mathscr{B}\left(\left(\lambda^{-1}\right)\right)^{4}$ and that, given a gauge invariant "vacuum" state $\psi_{0} \in \mathscr{B}\left(\left(\lambda^{-1}\right)\right)^{4}$, each element of $\mathscr{B}[\lambda]^{4}$ can be represented uniquely in the form $P \cdot \psi_{0}$. Then we can assign a scalar super Lax operator $L$ corresponding to a gauge equivalence class of the operator $\mathscr{L}$ by setting

$$
L \cdot \psi_{0}=\mu \psi_{0},
$$

where $\mu$ is a constant. The observation that $\Lambda^{4}$ is proportional to the unit matrix implies that $L$ is fourth order. The vacuum state $\psi_{0}$ is a constant vector annihilated by $F_{1}$ (the generator of $\mathfrak{n}$ ). We have two alternative choices of $\psi_{0}$,

$$
\begin{aligned}
& \psi_{B}=(1,-1,0,0)^{t}, \\
& \psi_{F}=(0,0,0,1)^{t} .
\end{aligned}
$$

The two choices are related by $\mathscr{L} \psi_{B}=2 \lambda \psi_{F}$.

We first consider the canonical gauge fixing

$$
\mathscr{L}^{\text {can }}=D+W \cdot F_{1}{ }^{2}+\Lambda .
$$

We have

$$
\begin{aligned}
& \left(\mathscr{L}^{\mathrm{can}}\right)^{4} \psi_{B}=\left(4 \lambda^{2},-4 \lambda^{2}, 0,-8 \lambda W\right)^{t}, \\
& \left(\mathscr{L}^{\mathrm{can}}\right)^{4} \psi_{F}=\left(4 W, 4 W, 0,-4 D W+4 \lambda^{2}\right)^{t} .
\end{aligned}
$$

Here we have taken into consideration the fact that $\Lambda$ appearing in $\mathscr{L}^{\text {can }}$ and $W$ are both Grassmann odd. We get from Eq. (4.16)

$$
\begin{aligned}
{\left[\left(\mathscr{L}^{\text {can }}\right)^{4}+4 W\left(\mathscr{L}^{\text {can }}\right)\right] \psi_{B} } & =4 \lambda^{2} \psi_{B}, \\
{\left[\left(\mathscr{L}^{\text {can }}\right)^{4}-4 W\left(\mathscr{L}^{\text {can }}\right)+4(D W)\right] \psi_{F} } & =4 \lambda^{2} \psi_{F} .
\end{aligned}
$$

The right-hand side of Eq. (4.17) are to be identified with $L_{B} \cdot \psi_{B}$ and $L_{F} \cdot \psi_{F}$, where

$$
\begin{aligned}
& L_{B}=D^{4}+4 W D, \\
& L_{F}=D^{4}-4 W D+4 D W .
\end{aligned}
$$

Manin and Radul constructed a possible form of super KP hierarchy and obtained the scalar Lax operator $L$ as a reduction of the hierarchy [10]. They found that the Lax equation

$$
\frac{d L}{d t}=\left[\left(L^{3 / 2}\right)^{+}, L\right]
$$

gives a supersymmetric extension of the $\mathrm{KdV}$ equation. The scalar super Lax operator $L_{B}$ constructed above coincides with their $L$ and it yields the equation

$$
\frac{d W}{d t}=\frac{1}{4} D^{6} W+3 D^{2} W D W+3 W D^{3} W .
$$


Equation (4.19) with $L=L_{F}$ gives the same equation.

Next we take the diagonal gauge fixing

$$
\mathscr{L}^{\text {diag }}=D+\Psi \cdot H+\Lambda .
$$

We note that by the identification $\Psi=D \Phi, \mathscr{L}^{\text {diag }}$ coinsides with the operator $\mathscr{L}$ of (4.5) for the super sine-Gordon equation. Noting Eq. (4.14), we readily find that

$$
\begin{aligned}
& \left(\mathscr{L}^{\text {diag }}-2 \Psi\right) \mathscr{L}^{\text {diag }}\left(\mathscr{L}^{\text {diag }}+2 \Psi\right) \mathscr{L}^{\text {diag }} \psi_{B}=4 \lambda^{4} \psi_{B}, \\
& \mathscr{L}^{\text {diag }}\left(\mathscr{L}^{\text {diag }}-2 \Psi\right) \mathscr{L}^{\text {diag }}\left(\mathscr{L}^{\text {diag }}+2 \Psi\right) \psi_{F}=4 \lambda^{4} \psi_{F} .
\end{aligned}
$$

Hence we have the super-differential operators of a factorized form,

$$
\begin{aligned}
& L_{B}=(D-2 \Psi) D(D+2 \Psi) D, \\
& L_{F}=D(D-2 \Psi) D(D+2 \Psi) .
\end{aligned}
$$

Since $\mathscr{L}^{\text {can }}$ and $\mathscr{L}^{\text {diag }}$ are gauge equivalent, we should identify the operators $L_{B}$ and $L_{F}$ of (4.18) with those of (4.23). This identification allows us to relate the dynamical variable $W$ in the canonical gauge fixing with the dynamical variable $\Psi$ in the diagonal gauge fixing as

$$
W=-\Psi D \Psi+\frac{1}{2} D^{2} \Psi .
$$

This relation is known as the super Miura transformation [16-18]. The super KdV equation (4.20) for $W$ is now transformed into the modified super $\mathrm{KdV}$ (msKdV) equation for $\Psi$,

$$
\frac{d \Psi}{d t}=\frac{1}{4} D^{6} \Psi-3(D \Psi)^{2} D^{2} \Psi-3 \Psi D \Psi D^{3} \Psi .
$$

In this section we have obtained the msKdV equation (4.25) from the scalar super Lax equation (4.19). Later we will show that the diagonal reduction of the super Lax type equation (3.14) with an appropriate choice of $\mathscr{A}$,

$$
\frac{d \mathscr{L}^{\text {diag }}}{d t}=\left[\mathscr{A}, \mathscr{L}^{\text {diag }}\right] \text {, }
$$

yields the msKdV equation.

\section{Conservation Laws}

In this section we derive the conservation laws for the generalized modified super $\mathrm{KdV}$ equation (3.14) proposed in Sect. 3. We begin by noting that in the bosonic case the conservation laws for Eq. (2.14) are derived by utilizing the adjoint action of $\Lambda$;

$$
\begin{aligned}
& \operatorname{ad} \Lambda: \mathrm{g}^{k} \rightarrow \mathrm{g}^{k+1}, \\
& \operatorname{ad} \Lambda(u)=[\Lambda, u] \text { for } u \in \mathrm{g}^{k} .
\end{aligned}
$$

The map ad $\Lambda$ has the following remarkable properties $[19,2]$ :

(i) $\mathfrak{g}$ admits a direct sum decomposition

$$
\mathfrak{g}=\operatorname{Ker} \operatorname{ad} \Lambda \oplus \operatorname{Im} \operatorname{ad} \Lambda .
$$


(ii) The subalgebra $\mathscr{Z}=\operatorname{Ker} \operatorname{ad} \Lambda$ is commutative.

These two properties are indispensable in constructing the conservation laws for the Lax type equation (2.14). The decomposition (5.2) allows us to separate the gauge degrees of freedom related to the gauge transformation (2.10) from the physical conservation laws. The conservation laws are elements of $\operatorname{Ker} \operatorname{ad} \Lambda$.

To construct the conservation laws in the supersymmetric case we need a map which yields a direct sum decomposition of the affine Lie superalgebra $g$ analogous to Eq. (5.2). $\Lambda$ is a Grassmann odd element of $\mathfrak{g}$ and we readily see that neither of the two properties (i) and (ii) holds; the element $\Lambda^{2}=\frac{1}{2}[\Lambda, \Lambda]_{+}$belongs to both $\operatorname{Ker}$ ad $\Lambda$ and $\operatorname{Im}$ ad $\Lambda$. Regarding (ii), in general the anti-commutator of two odd elements of $\operatorname{Ker}$ ad $\Lambda$ is a non-vanishing element of $\operatorname{Ker}$ ad $\Lambda$. After some exercise we have found that for a wide class of affine Lie superalgebra, ad $\Lambda^{2}$ has the property (i); ad $\Lambda^{2}$ gives the direct sum decomposition

$$
\mathfrak{g}=\operatorname{Ker} \operatorname{ad} \Lambda^{2} \oplus \operatorname{Im} \operatorname{ad} \Lambda^{2} \text {. }
$$

We have proved the relation (5.3) for $\operatorname{OSp}(2 \mid 2)^{(2)}$ by explicit computation. For $S L(n \mid n)^{(1)}$ the proof can be made by reducing the problem to that of proving the relation (5.2) for bosonic affine Lie algebras [20]. In this paper we will deal with the super Lax type equation (3.14) associated with those affine Lie superalgebras which admit the direct sum decomposition (5.3) in terms of ad $\Lambda^{2}$.

In accordance with the decomposition (5.3) using ad $\Lambda^{2}$ in place of ad $\Lambda$, we are led to consider the square of the original super Lax operator $\mathscr{L}$ of (3.13),

$$
\mathscr{L}^{2}=\frac{\partial}{\partial x}+D q+\frac{1}{2}[q, q]_{+}+[\Lambda, q]_{+}+\Lambda^{2} .
$$

Note that the time evolution of $\mathscr{L}^{2}$ is governed by the same operator $\mathscr{A}$ that is used in (3.14);

$$
\frac{d \mathscr{L}^{2}}{d t}=\left[\mathscr{A}, \mathscr{L}^{2}\right]
$$

Thanks to the decomposition (5.3), we can prove the following proposition.

Proposition 5.1. There exists an element $U=\sum_{i<0} U^{i}$ with $U^{i} \in \mathfrak{g}^{i}$, such that the gauge transformation by $U$ takes $\mathscr{L}^{2}$ into the form ${ }^{i<0}$

$$
\mathscr{L}_{0}^{2}:=\exp (\operatorname{ad} U) \cdot \mathscr{L}^{2}=\frac{\partial}{\partial x}+Q_{0}+\Lambda^{2} .
$$

Here $Q_{0}=\sum_{k \leqq 0} Q_{0}{ }^{k}$ and each component $Q_{0}{ }^{k}$ belongs to $\mathrm{g}^{k} \cap \operatorname{Ker} \operatorname{ad} \Lambda^{2}$.

Proof. By taking the $\mathrm{g}^{k}$-component of (5.6), we see that $Q_{0}{ }^{k}+\left[\Lambda^{2}, U^{k-2}\right]$ is expressed as a differential polynomial in $Q_{0}{ }^{i}(k<i), U^{j}(k-2<j)$ and the components of $q$. Using the decomposition (5.3), we can determine $U$ and $Q_{0}$ inductively as differential polynomials in the components of $q$.

The gauge transformation by $U$ takes the time evolution equation (5.5) into the form

$$
\frac{\partial Q_{0}}{\partial t}+\frac{\partial \tilde{\mathscr{A}}}{\partial x}=\left[\tilde{\mathscr{A}}, Q_{0}+\Lambda^{2}\right]
$$


where $\tilde{\mathscr{A}}$ is the gauge transform of $\mathscr{A}$,

$$
\partial / \partial t-\tilde{A}=\exp \operatorname{ad} U(\partial / \partial t-\mathscr{A}) .
$$

Lemma 5.2. $\tilde{A}$ belongs to $\operatorname{Ker} \operatorname{ad} \Lambda^{2}$.

Proof. Let us set $\tilde{\mathscr{A}}=\sum_{k \leqq n} A^{k}$ with $A^{k} \in \mathfrak{g}^{k}$. The $\mathrm{g}^{k}$-component of (5.7) gives

$$
\frac{\partial Q_{0}{ }^{k}}{\partial t}+\frac{\partial A^{k}}{\partial x}-\sum_{i+j=k}\left[A^{i}, Q_{0}{ }^{j}\right]=\left[A^{k-2}, \Lambda^{2}\right] \text {. }
$$

Suppose $A^{i}(k-1 \leqq i \leqq n)$ belongs to $\operatorname{Ker}$ ad $\Lambda^{2}$. Then the left-hand side of (5.9) is in $\operatorname{Ker}$ ad $\Lambda^{2}$, while the right-hand side is in $\operatorname{Im}$ ad $\Lambda^{2}$. By the assumption (5.3), both side of (5.9) must vanish. Hence, by induction, $\left[\Lambda^{2}, \tilde{\mathscr{A}}\right]=0$.

\section{Corollary 5.3.}

$$
\frac{\partial Q_{0}{ }^{k}}{\partial t}+\frac{\partial A^{k}}{\partial x}=\sum_{i+j=k}\left[A^{i}, Q_{0}^{j}\right] .
$$

In the bosonic case the right-hand side of the equation corresponding to Eq. (5.10) vanishes, because the subalgebra $\operatorname{Ker}$ ad $\Lambda$ is abelian, implying that each component $Q_{0}{ }^{k}$ is a conserved current. In the present case the right-hand side of (5.10) does not vanish in general. To obtain conservation laws from Eq. (5.10) we need the following trick of "abelianization" of $\mathscr{K}=\operatorname{Ker}$ ad $\Lambda^{2}$. Let $[\mathscr{K}, \mathscr{K}]$ denote the commutant of $\mathscr{K}$, subalgebra generated by elements of the form $[k, l](k, l \in \mathscr{K})$. In terms of a certain Killing form we define the orthogonal complement of $[\mathscr{K}, \mathscr{K}]$ in $\mathscr{K}$ denoted by $\widetilde{K} . \widetilde{K}$ is isomorphic to the quotient algebra $\mathscr{K} /[\mathscr{K}, \mathscr{K}] . \mathscr{K}$ is decomposed as

$$
\mathscr{K}=[\mathscr{K}, \mathscr{K}] \oplus \tilde{\mathscr{K}} .
$$

Since the right-hand side of $(5.10)$ is an element of $[\mathscr{K}, \mathscr{K}]$, the $\tilde{K}$-component of (5.10) vanishes and hence it gives a conservation law. Therefore, if $\tilde{K} \cap g^{k}$ is non-trivial we have a conserved current of grade $k$. Note that the set $\mathscr{E}:=\{k \in Z$; $\left.\tilde{\mathscr{K}} \cap \mathrm{g}^{k} \neq 0\right\}$ is determined from the commutation relations and that there exists a period $n$ such that $\mathscr{E}+n=\mathscr{E}$. This implies the existence of infinitely many conserved quantities. In the bosonic case the period is called (dual) Coxeter number.

We illustrate our construction of conservation laws in the case of $O S p(2 \mid 2)^{(2)}$, the example discussed in Sect. 4. As worked out in Appendix $B, \mathscr{K}$ is spanned by

$$
\begin{array}{ll}
\lambda^{2 j} \Lambda \in \mathfrak{g}^{1+4 j}, & \lambda^{2 j}\left(F_{1}-F_{0}\right) \in \mathfrak{g}^{-1+4 j}, \\
\lambda^{-2+2 j} \Lambda^{2}, & \lambda^{2 j} \bar{\Lambda}^{2} \in \mathfrak{g}^{-2+4 j},
\end{array}
$$

and the subspace $\tilde{\mathscr{K}}$ is spanned by

$$
\lambda^{2 j} \bar{\Lambda}^{2} \in \mathfrak{g}^{-2+4 j} \text {. }
$$

Hence $\tilde{K} \cap \mathfrak{g}^{k} \neq 0$ if and only if $k \equiv 2(\bmod .4$, corresponding to period $n=4)$. This means that the conservation laws belong to $Q_{0}^{-2+4 j}$.

We have computed the first few terms of $Q_{0}{ }^{k}(k \leqq 0)$ by taking the diagonal reduction

$$
q^{\mathrm{diag}}=D \Phi \cdot H
$$


corresponding to the super Lax operator (4.5) for the super sine-Gordon equation. The operator $\mathscr{L}^{2}$ is given by

where

$$
\mathscr{L}^{2}=\frac{\partial}{\partial x}+Q^{0}+Q^{1}+\Lambda^{2}
$$

$$
\begin{aligned}
& Q^{0}=D^{2} \Phi \cdot H, \\
& Q^{1}=D \Phi \cdot\left(E_{1}-E_{0}\right) .
\end{aligned}
$$

The components $Q_{0}{ }^{k}$ and $U^{k}$ are obtained inductively as differential polynomials in $\Phi$ by solving the relation (5.6). The first few terms are given in Appendix C. The first two conservation laws are obtained as the coefficients of $Q_{0}^{-2}$ and $Q_{0}^{-6}$ and are found to be

$$
\begin{aligned}
& J^{1}=-\frac{1}{4} D[\partial \Phi D \Phi], \\
& J^{2}=-\frac{1}{16} D\left[\partial \Phi D^{5} \Phi-D \Phi(\partial \Phi)^{3}\right] .
\end{aligned}
$$

Their integrations

$$
\begin{aligned}
& Q^{(3 / 2)}=-\frac{1}{4} \int d x J^{1}(x)=-\frac{1}{4} \int d x d \theta(\partial \Phi D \Phi), \\
& Q^{(7 / 2)}=-\frac{1}{16} \int d x J^{2}(x)=-\frac{1}{16} \int d x d \theta\left(\partial \Phi D^{5} \Phi-D \Phi(\partial \Phi)^{3}\right),
\end{aligned}
$$

reproduce the conserved charges obtained earlier in non-Lie algebraic methods $[15,21]$.

\section{Consistent Time Flow Generators}

In the super Lax type equation (3.14), the dynamical variable $q$ in $\mathscr{L}$ is assumed to be $\mathfrak{b}$-valued. A consistent time evolution requires that the commutator $[\mathscr{A}, \mathscr{L}]$ be also $\mathfrak{b}$-valued. We will show how to construct generators $\mathscr{A}$ such that $[\mathscr{A}, \mathscr{L}]$ is $\mathfrak{b}$-valued.

Let us start with the following lemma.

Lemma 6.1. Let $M=\sum_{k=-\infty}^{n} M^{k}\left(M^{k} \in \mathrm{g}^{k}\right)$ be an (even) superfield. If $[M, \mathscr{L}]=0$, then $\left[M^{+}, \mathscr{L}\right]$ is b-valued, where $M^{+}=\sum_{k=0}^{n} M^{k}$.

Proof. $[M, \mathscr{L}]=0$ implies

$$
\left[M^{+}, \mathscr{L}\right]=-\left[M^{-}, \mathscr{L}\right],
$$

where $M^{-}=M-M^{+}$. We must prove that $\left[M^{+}, \mathscr{L}\right]$ belongs to both $\mathfrak{g}_{0}$ and $\bigoplus_{k \leqq 0} \mathfrak{g}^{k}$ (see Eq. (2.3)). We see that because of the property $\left[\mathfrak{g}^{k}, \mathfrak{g}^{l}\right] \subset \mathrm{g}^{k+l}$ of the principal (canonical) gradation $\left[M^{-}, \mathscr{L}\right]$ does not have components with a positive grade. To be convinced that $\left[\mathrm{M}^{+}, \mathscr{L}\right]$ belongs to $\mathrm{g}_{0}$, one notes that there exists a positive integer $n$ such that $\bigoplus_{-n \leqq k \leqq n} \mathfrak{g}^{k} \subset g_{0} \subset \bigoplus_{-(n+1) \leqq k \leqq(n+1)} \mathfrak{g}^{k}$.

Because of this lemma the problem is reduced to constructing superfields $M$ which commute with $\mathscr{L}$. A family of such superfields can be obtained from elements 
of $Z(\mathscr{K})$, the center of $\operatorname{Ker} a d \Lambda^{2}$, by means of the inverse gauge transformation by $U=\sum_{i<0} U^{i}$ which appeared in the last section (see (5.6)).

Proposition 6.2. Let $M(c)=\exp (-\operatorname{ad} U) \cdot c$, where $c$ is an (even) element of $Z(\mathscr{K})$, then $[M(c), \mathscr{L}]=0$.

Proof. It is sufficient to show $\left[c, \mathscr{L}_{0}\right]=0$, where we have defined $\mathscr{L}_{0}$ by

$$
\mathscr{L}_{0}:=\exp (\operatorname{ad} U) \mathscr{L} \text {. }
$$

Write $\mathscr{L}_{0}$ in the form

$$
\mathscr{L}_{0}=D+R+\Lambda \text {. }
$$

Since $\Lambda$ belongs to $\operatorname{Ker}$ ad $\Lambda^{2}$, the proof is completed by proving the following lemma.

Lemma 6.3. $R$ belongs to $\operatorname{Ker}$ ad $\Lambda^{2}$.

Proof. Since $U$ does not contain components with positive grade, the odd superfield $R$ has no positively graded components, hence $R=\sum_{k \leqq 0} R^{k}$. Comparing (6.3) with (5.6), we obtain the relation of $R$ and $Q_{0}$,

$$
Q_{0}=\frac{1}{2}[R, R]_{+}+D R+[\Lambda, R]_{+} .
$$

Let us first show that $R^{0}=0$. The defining relation (6.2) gives

$$
R^{0}=q^{0}+\left[U^{-1}, \Lambda\right]
$$

On the other hand, $\mathfrak{g}^{1}$-component of Eq. (5.6) tells

$$
\left[U^{-1}, \Lambda^{2}\right]+\left[\Lambda, q^{0}\right]=0 .
$$

Hence we have

$$
\left[\Lambda, R^{0}\right]=0 .
$$

This holds if and only if $R^{0}$ is proportional to the central element, which we can set zero. Then $\mathfrak{g}^{-k}$-component of (6.4) is

$$
Q_{0}^{-k}=D R^{-k}+\left[\Lambda, R^{-k-1}\right]_{+}+\frac{1}{2} \sum_{i+j=k}\left[R^{-i}, R^{-j}\right]_{+} .
$$

To use induction, suppose all $R^{-i}(i=0,1, \ldots, k)$ are in $\operatorname{Ker} a d \Lambda^{2}$, then we easily see that all terms in (6.8) except $\left[\Lambda, R^{-k-1}\right]$ belong to $\operatorname{Ker}$ ad $\Lambda^{2}$. Hence $\left[\Lambda, R^{-k-1}\right] \epsilon$ $\operatorname{Ker}$ ad $\Lambda^{2}$. From the Jacobi identity we have

$$
\begin{gathered}
{\left[\Lambda, \operatorname{Ker} \operatorname{ad} \Lambda^{2}\right] \subset \operatorname{Ker} \operatorname{ad} \Lambda^{2},} \\
{\left[\Lambda, \operatorname{Im} \operatorname{ad} \Lambda^{2}\right] \subset \operatorname{Im} \operatorname{ad} \Lambda^{2} .}
\end{gathered}
$$

Since $\operatorname{Ker}$ ad $\Lambda^{2} \cap \operatorname{Im}$ ad $\Lambda^{2}=\{0\},\left[\Lambda, R^{-k-1}\right] \in \operatorname{Ker}$ ad $\Lambda^{2}$ implies $R^{-k-1} \in \operatorname{Ker}$ ad $\Lambda^{2}$. Thus we have proved that $R$ belongs to $\operatorname{Ker}$ ad $\Lambda^{2}$.

Combining Lemma 6.1 and Proposition 6.2, we see that

$$
\frac{\partial \mathscr{L}}{\partial t}=\left[M(c)^{+}, \mathscr{L}\right] \quad(c \in Z(\mathscr{K}))
$$

defines a family of consistent time evolution equations. We note that $\Lambda^{2}=\frac{1}{2}[\Lambda, \Lambda]_{+}$ 
is a non-trivial element of $Z(\mathscr{K})$ and hence Eq. (6.10) is not empty. In parallel with our derivation of conserved currents in the previous section, the set $\mathscr{E}^{\prime}:=\left\{k \in \mathbf{Z} ; Z(k) \cap \mathfrak{g}^{k} \neq 0\right\}$ has the period $\boldsymbol{n}$. (Presumably $\mathscr{E}^{\prime}$ coincides with $\mathscr{E}$ in Sect. 5.) Therefore, Eq. (6.10) gives an infinite series of the time evolution equation. In the $\operatorname{OSp}(2 \mid 2)^{(2)}$ case to be discussed below, $\Lambda^{4 k+2}=(2 \lambda)^{2 k} \Lambda^{2}(k \in \mathbf{Z})$ span $Z(\mathscr{K})$. We can also check the hierarchy structure in the case of $S L(n \mid n)^{(1)}$. By taking the standard $(2 n) \times(2 n)$ matrix representation, it is easy to show that $Z(\mathscr{K})$ is spanned by $\left.\Lambda^{2 k}(k \equiv 0 \bmod . n)\right), \Lambda^{2 n}$ being proportional to the identity.

The generalized super $\mathrm{KdV}$ equations (6.10) can be put into a simpler form in the diagonal gauge fixing. Set

$$
q^{\mathrm{diag}}=\sum_{a=1}^{r} Q_{a} H_{a} .
$$

By taking the 0-th grade component on both sides of (6.10), we get

$$
\sum_{a=1}^{r}\left(\partial Q_{a} / \partial t\right) \cdot H_{a}=-D(M(c))^{0} .
$$

We apply the present method to the case of $O S p(2 \mid 2)^{(2)}$ and show that the super KdV equation given by Manin and Radul arises in the hierarchy (6.10). We see that $\Lambda^{4 k+2}=(2 \lambda)^{2 k} \Lambda^{2} \in \mathfrak{g}^{4 k+2}$ and these elements span $Z(\mathscr{K})$. We take the diagonal gauge fixing

$$
\mathscr{L}^{\mathrm{diag}}=D+\Psi \cdot H+\Lambda .
$$

The hierarchy (6.12) takes the form

$$
\frac{\partial \Psi}{\partial t} \cdot H=-D\left(M_{k}\right)^{0}
$$

where $M_{k}:=\exp (-\operatorname{ad} U) \cdot \Lambda^{4 k+2}(k \geqq 0)$. Consider the case of $k=1 . M_{1}$ is computed in Appendix D.

$$
\begin{aligned}
\left(M_{1}\right)^{0} & =\left[\exp (-\operatorname{ad} U) \cdot \Lambda^{6}\right]^{0} \\
& =\left[D^{5} \Psi-2(D \Psi)^{3}+3 \Psi D \Psi \partial \Psi\right] \cdot H .
\end{aligned}
$$

Thus Eq. (6.14) for $k=1$ gives

$$
\frac{\partial \Psi}{\partial t}=-\partial^{3} \Psi+3 \partial \Psi(D \Psi)^{2}+3 \Psi D \Psi D^{3} \Psi,
$$

which coincides with the modified super $\mathrm{KdV}$ equation (4.25) derived from the scalar Lax equation.

\section{Discussion}

Our construction of an infinite series of conservation laws and the consistent time evolution generators for the generalized modified super $\mathrm{KdV}$ hierarchy relies on the direct sum decomposition (5.3) of the affine Lie superalgebra in terms of ad $\Lambda^{2}$. In the bosonic case the analogous construction relies on the decomposition (5.2) in terms of ad $\Lambda$, which is proved to be true for an arbitrary affine Lie algebra 
$[19,2]$. We feel that the relation (5.3) is a basic property of those affine Lie superalgebras which admit a superprincipal embedding of $\operatorname{OSp}(1 \mid 2)$, and hence it holds true for the affine Lie superalgebras listed in (3.5). Presently we have proved (5.3) for $S L(n \mid n)^{(1)}$ and $\operatorname{OSp}(2 \mid 2)^{(2)}$.

The generalized (modified) super $\mathrm{KdV}$ equations we have proposed are in a Lax type representation. There is a folklore that those nonlinear differential equations which have a Lax type representation are integrable. We believe that our generalized (modified) super $\mathrm{KdV}$ equations give supersymmetric integrable systems. The existence of an infinite series of conserved currents discussed in Sect. 5 lends a support to our belief. The integrability can be proved by showing further that conserved currents are mutually commuting and that there exist sufficiently many infinite series of conserved currents. To complete the proof we need better understanding of the properties of the map ad $\Lambda^{2}$ of Lie superalgebras.

The time coordinate which appears in the super Lax type equation (3.14) is Grassmann even. Accordingly the time flow generators and the conservation laws are all even. The physical meaning of conservation laws with respect to Grassmann odd time is obscure to us and we have not considered the odd time evolution of the super Lax operator $\mathscr{L}$ in this paper. One may introduce odd time $\tau$ and the associated superderivative $D_{\tau}:=\partial / \partial \tau+\tau \partial / \partial t$ with the property $D_{\tau}^{2}=\partial / \partial t$. The super Lax type equation

$$
D_{\tau} \mathscr{L}=[\mathscr{A}, \mathscr{L}]_{+}
$$

will possibly allow us to study odd time evolution as well as even time evolution. We should note that a reduction of the super KP hierarchy of Manin and Radul yields both even and odd time evolution. Presumably the consistent time flow generators for Eq. (7.1) can be constructed by extending the method given in Sect. 6 .

A hierarchy of generalized $\mathrm{KdV}$ equations is known to be obtained as a reduction of the KP hierarchy [22]. The latter hierarchy is related to the deformation problem of the pseudo-differential operator $\mathscr{L}=\partial+u_{0}+u_{1} \partial^{-1}+$ $u_{2} \partial^{-2}+\cdots$. In the Lie algebraic approach the KP hierarchy is obtained by considering the Lax type equation associated with $\left.G L(n)^{(1)}\right|_{n \rightarrow \infty}$. The Lie superalgebraic method proposed in this paper applies to the super Lax type equation associated with $S L(n \mid n)^{(1)}$. It is an interesting problem whether a super KP hierarchy is obtained by considering the $n \rightarrow \infty$ limit of the super Lax operator associated with $S L(n \mid n)^{(1)}$. This approach will help clarify the connection between the different schemes of super KP hierarchy recently proposed [10,23].

$W_{n}$ algebras are known to be obtained from the second Hamiltonian structure of the scalar Lax equation (2.32) or equivalently the Lax type equation (2.14) for the gauge fixed Lax operator $\mathscr{L}[2,3]$; they are contained in the second Gel'fand-Dikii (G-D) brackets [24]. A supersymmetric generalization of the G-D bracket for generalized super $\mathrm{KdV}$ equations will enable us to construct super $W_{n}$ algebras in an analogous way.

We briefly sketch how the super $\mathrm{G}-\mathrm{D}$ bracket may be constructed starting from a supersymmetric current algebra. Let $\Psi^{a}(z, \theta)=\psi^{a}(z)+\theta j^{a}(z)$ be an $n$-component odd superfield of weight $\frac{1}{2}, j^{a}(z)$ being a bosonic current. We assume that $\Psi^{a}$ satisfies the following type of current algebra:

$$
\left\{\Psi^{a}\left(z_{1}, \theta_{1}\right), \Psi^{b}\left(z_{2}, \theta_{2}\right)\right\}=C^{a b} D_{1}\left[\delta\left(z_{1}-z_{2}\right)\left(\theta_{1}-\theta_{2}\right)\right]
$$


where $C^{a b}$ is a constant determined from the structure of the Lie superalgebra under consideration. We introduce superfields $U_{k}$ of weight $(n-k) / 2$ by assuming a generalized super Miura transformation

$$
D^{n}+U_{n-1} D^{n-1}+\cdots+U_{1} D+U_{0}=\left(D-\Psi^{1}\right)\left(D-\Psi^{2}\right) \cdots\left(D-\Psi^{n}\right) .
$$

The bracket structure of $U_{k}$ induced from (7.2) defines the super G-D algebra.

The simplest example of this construction appears in connection with the super Miura transformation discussed in Sect. 4,

$$
D^{4}+4 W D=(D-2 \Psi) D(D+2 \Psi) D .
$$

Assume that $\Psi$ satisfies the $U(1)$ current algebra

$$
\left\{\Psi\left(z_{1}, \theta_{1}\right), \Psi\left(z_{2}, \theta_{2}\right)\right\}=D_{1}\left[\delta\left(z_{1}-z_{2}\right)\left(\theta_{1}-\theta_{2}\right)\right] \text {. }
$$

The modified super $\mathrm{KdV}$ equation for $\Psi$, Eq. (4.25), is written as the Hamiltonian equation using the Poisson bracket (7.5). The super G-D bracket for $W$ is obtained from the bracket (7.5) and it gives the super Virasoro algebra of the super stress tensor $W$, as previously pointed out [17].

Super $W_{n}$ algebras can also be constructed by computing operator product expansions of currents of higher spin and demanding associativity [25]. This program is difficult to extend to a general case. The derivation of super $W_{n}$ algebras from the second Hamiltonian structure of generalized super $\mathrm{KdV}$ equations sketched above appears to provide a more systematic way of constructing super $W_{n}$ algebras.

\section{Appendix. $O S p(2 \mid 2)^{(2)}$ and Modified Super KdV Equation}

A. $\operatorname{Osp}(2 \mid 2)^{(2)}$. Two inequivalent Dynkin graphs, a and b of Fig. 1, correspond to the Lie superalgebra $G=O S p(2 \mid 2)$. We take the Dynkin graph 1 b corresponding to a purely fermionic SRS. The Chevalley generators in this basis satisfy the commutation relations

$$
\begin{array}{ll}
{\left[h_{a}, h_{b}\right]=0,} & {\left[e_{a}, f_{b}\right]_{+}=\delta_{a b} h_{b},} \\
{\left[h_{a}, e_{b}\right]=K_{a b} e_{b},} & {\left[h_{a}, f_{b}\right]=-K_{a b} f_{b},}
\end{array}
$$

where the Cartan matrix is

$$
K_{a b}=\left(\begin{array}{ll}
0 & 2 \\
2 & 0
\end{array}\right) .
$$

One can define a $Z_{2}$ automorphism of $G$ associated with the $Z_{2}$ symmetry of the Dynkin graph $1 \mathrm{~b}$ regarding the exchange $\left\{e_{1}, f_{1}\right\} \leftrightarrow\left\{e_{2}, f_{2}\right\}$. $G$ is decomposed into the even and odd part, $G=G_{\overline{0}} \oplus G_{\overline{1}}$, under this involution. $G_{\overline{0}}$ consists of the fixed points in $G$. It is $(3,2)$-dimensional and isomorphic to $O S p(1 \mid 2)$. $G_{\overline{1}}$ satisfies $\left[G_{\overline{0}}, G_{\overline{1}}\right] \subset G_{\overline{1}}$, i.e., $G_{\overline{1}}$ is a $G_{\overline{0}}$-module of dimension $(1,2)$, and it provides a representation of $G_{\overline{0}} \cong O S p(1 \mid 2)$. We define the twisted affine Lie superalgebra $\mathfrak{g}=\operatorname{OSp}(2 \mid 2)^{(2)}$ to have the SRS given by adding the lowest weight of $G_{\overline{0}}$-module $G_{\overline{1}}$ to the SRS of $G_{\overline{0}}$.

$$
E_{1}=e_{1}+e_{2}, \quad E_{0}=\lambda\left(f_{1}-f_{2}\right)
$$




$$
\begin{aligned}
& F_{1}=f_{1}+f_{2}, \quad F_{0}=\lambda^{-1}\left(e_{1}-e_{2}\right), \\
& H_{1}=H_{0}=h_{1}+h_{2}=H .
\end{aligned}
$$

The spectral parameter $\lambda$ is introduced to realize $\operatorname{OSp}(2 \mid 2)^{(2)}$ as a subalgebra of $\operatorname{OSp}(2 \mid 2) \otimes C\left[\lambda, \lambda^{-1}\right]$. The commutation relations of the generators $\left\{E_{a}, F_{a}, H_{a}\right\}_{a=0}^{1}$ can be calculated from Eq. (A.1) and they are

$$
\begin{aligned}
& {\left[H_{a}, H_{b}\right]=0, \quad\left[E_{a}, F_{b}\right]_{+}=\delta_{a b} H_{b},} \\
& {\left[H_{a}, E_{b}\right]=K_{a b} E_{b}, \quad\left[H_{a}, F_{b}\right]=-K_{a b} F_{b},}
\end{aligned}
$$

where the Cartan matrix is

$$
K_{a b}=\left(\begin{array}{rr}
2 & -2 \\
-2 & 2
\end{array}\right) .
$$

The corresponding Dynkin graph is shown in Fig. 2.

To introduce the super $\mathscr{D}$-module structure in Sect. 4 we need a matrix representation of the generators of $\operatorname{OSP}(2 \mid 2)^{(2)}$. We take the following $4 \times 4$ matrix representation of $O S p(2 \mid 2)$ in which we have

$$
\begin{aligned}
& e_{1}=\left(\begin{array}{cccc}
0 & 0 & 0 & 0 \\
0 & 0 & 0 & 1 \\
1 & 0 & 0 & 0 \\
0 & 0 & 0 & 0
\end{array}\right), \quad e_{2}=\left(\begin{array}{llll}
0 & 0 & 0 & 1 \\
0 & 0 & 0 & 0 \\
0 & 1 & 0 & 0 \\
0 & 0 & 0 & 0
\end{array}\right), \\
& f_{1}=\left(\begin{array}{rrrr}
0 & 0 & 1 & 0 \\
0 & 0 & 0 & 0 \\
0 & 0 & 0 & 0 \\
0 & -1 & 0 & 0
\end{array}\right), \quad f_{2}=\left(\begin{array}{rrrr}
0 & 0 & 0 & 0 \\
0 & 0 & 1 & 0 \\
0 & 0 & 0 & 0 \\
-1 & 0 & 0 & 0
\end{array}\right), \\
& h_{1}=\left(\begin{array}{rrrr}
1 & 0 & 0 & 0 \\
0 & -1 & 0 & 0 \\
0 & 0 & 1 & 0 \\
0 & 0 & 0 & -1
\end{array}\right), \quad h_{2}=\left(\begin{array}{rrrr}
-1 & 0 & 0 & 0 \\
0 & 1 & 0 & 0 \\
0 & 0 & 1 & 0 \\
0 & 0 & 0 & -1
\end{array}\right) \text {, }
\end{aligned}
$$

and hence

$$
\begin{aligned}
& E_{1}=\left(\begin{array}{llll}
0 & 0 & 0 & 1 \\
0 & 0 & 0 & 1 \\
1 & 1 & 0 & 0 \\
0 & 0 & 0 & 0
\end{array}\right), \quad E_{0}=\left(\begin{array}{rrrr}
0 & 0 & \lambda & 0 \\
0 & 0 & -\lambda & 0 \\
0 & 0 & 0 & 0 \\
\lambda & -\lambda & 0 & 0
\end{array}\right), \\
& F_{1}=\left(\begin{array}{rrrr}
0 & 0 & 1 & 0 \\
0 & 0 & 1 & 0 \\
0 & 0 & 0 & 0 \\
-1 & -1 & 0 & 0
\end{array}\right), \quad F_{0}=\left(\begin{array}{cccc}
0 & 0 & 0 & -\lambda^{-1} \\
0 & 0 & 0 & \lambda^{-1} \\
\lambda^{-1} & -\lambda^{-1} & 0 & 0 \\
0 & 0 & 0 & 0
\end{array}\right) \text {, } \\
& H=\left(\begin{array}{rrrr}
0 & 0 & 0 & 0 \\
0 & 0 & 0 & 0 \\
0 & 0 & 2 & 0 \\
0 & 0 & 0 & -2
\end{array}\right)
\end{aligned}
$$


Fig. 2. Dynkin graph of the twisted affine Lie superalgebra $\operatorname{OSp}(2 \mid 2)^{(2)}$

$B$. Ker ad $\Lambda^{2}$. Define $\Lambda$ and $\bar{\Lambda}$ as the sum of generators of positive and negative SRS of $\operatorname{OSp}(2 \mid 2)^{(2)}$.

$$
\Lambda=E_{0}+E_{1}, \quad \bar{\Lambda}=F_{0}+F_{1} .
$$

We show the diagonal sum decomposition (5.3) of $\operatorname{OSp}(2 \mid 2)^{(2)}$ by explicit computation of

$$
\operatorname{ad} \Lambda^{2}: \mathrm{g}^{k} \rightarrow \mathrm{g}^{k+2} .
$$

The $\mathscr{K}:=\operatorname{Ker} \operatorname{ad} \Lambda^{2}$ is spanned by

$$
\begin{gathered}
\lambda^{2 j} \Lambda \in \mathfrak{g}^{+1+4 j}, \\
\lambda^{2 j}\left(F_{1}-F_{0}\right) \in \mathfrak{g}^{-1+4 j}, \\
\lambda^{-2+2 j} \Lambda^{2}, \quad \lambda^{2 j} \bar{\Lambda}^{2} \in \mathfrak{g}^{-2+4 j} .
\end{gathered}
$$

The $\mathscr{K}^{\perp}=\operatorname{Im}$ ad $\Lambda^{2}$ is spanned by

$$
\begin{aligned}
& \lambda^{2 j}\left(E_{1}-E_{0}\right) \in \mathfrak{g}^{+1+4 j}, \\
& \lambda^{2 j} H \in \mathfrak{g}^{4 j}, \\
& \lambda^{2 j} \bar{\Lambda} \in \mathfrak{g}^{-1+4 j}, \\
& \lambda^{2 j}\left(F_{1}^{2}-F_{0}^{2}\right) \in \mathfrak{g}^{-2+4 j} .
\end{aligned}
$$

We see from (A.10) and (A.11) that the direct sum decomposition

$$
\mathfrak{g}^{k}=\mathscr{K}^{k} \oplus\left(\mathscr{K}^{\perp}\right)^{k}
$$

holds.

The $\mathscr{K}$ is further decomposed as

$$
\mathscr{K}=[\mathscr{K}, \mathscr{K}] \oplus \tilde{\mathscr{K}} .
$$

Here the commutant $[\mathscr{K}, \mathscr{K}]$ is spanned by

$$
\begin{aligned}
& \lambda^{2 j} \Lambda \in \mathfrak{g}^{1+4 j}, \\
& \lambda^{2 j}\left(F_{1}-F_{0}\right) \in \mathfrak{g}^{-1+4 j}, \\
& \lambda^{-2+2 j} \Lambda^{2} \in \mathfrak{g}^{-2+4 j} .
\end{aligned}
$$

The orthogonal complement $\tilde{\mathscr{K}}$ is spanned by

$$
\lambda^{2 j} \bar{\Lambda}^{2} \in \mathfrak{g}^{-2+4 j}
$$

i.e., only $\tilde{K}^{-2+4 j}$ are non-empty.

C. Conservation Laws. The gauge transformation by $U$ and the term $Q_{0}$ in $\left(\mathscr{L}^{2}\right)_{0}$ are obtained by solving

$$
\begin{aligned}
\partial+\sum_{i=1}^{\infty}\left(Q_{0}\right)^{-i}+\Lambda^{2}= & e^{\operatorname{ad} U}\left(\mathscr{L}^{2}\right) \\
= & \partial+Q^{0}+Q^{1}+\Lambda^{2}+\left[U, \partial+Q^{0}+Q^{1}+\Lambda^{2}\right] \\
& +\frac{1}{2}\left[U,\left[U, \partial+Q^{0}+Q^{1}+\Lambda^{2}\right]\right] \\
& +\frac{1}{6}\left[U,\left[U,\left[U, \partial+Q^{0}+Q^{1}+\Lambda^{2}\right]\right]\right]+\cdots,
\end{aligned}
$$


iteratively. Here $Q^{0}=D \Psi \cdot H$ and $Q^{1}=\Psi \cdot\left(E_{1}-E_{0}\right)$. The first few terms of $U^{-i}$ and $\left(Q_{0}\right)^{-i}$ are

$$
\begin{aligned}
U^{-1}= & -\frac{1}{2} \Psi \bar{\Lambda}+x \Psi\left(F_{1}-F_{0}\right), \\
U^{-2}= & -\frac{1}{2} D \Psi\left(F_{1}^{2}-F_{0}^{2}\right)+D \Psi\left(y \bar{\Lambda}^{2}-z \lambda^{-2} \Lambda^{2}\right), \\
U^{-3}= & -\frac{1}{4}[\partial \Psi+(x+2 z) \Psi D \Psi] \lambda^{-2}\left(E_{1}-E_{0}\right) \\
& +(u \partial \Psi+v \Psi D \Psi) \lambda^{-2} \Lambda, \\
U^{-4}= & -\frac{1}{4}\left[\partial D \Psi+2(y+z)(D \Psi)^{2}-(x-2 u) \Psi \partial \Psi\right] \lambda^{-2} H, \\
\vdots & \\
Q_{0}^{-1}= & -\left[\partial(x \Psi)+\frac{1}{2} \Psi D \Psi\right]\left(F_{1}-F_{0}\right), \\
Q_{0}^{-2}= & -\left[\partial(y D \Psi)+\frac{1}{4}(D \Psi)^{2}-\frac{1}{4} \Psi \partial \Psi\right] \bar{\Lambda}^{2} \\
& +\left[\partial(z D \Psi)+\frac{1}{4}(D \Psi)^{2}-x \Psi \partial(x \Psi)\right] \lambda^{-2} \Lambda^{2}, \\
Q_{0}^{-3}= & {\left[\frac{1}{4} \partial(u \partial \Psi)+\frac{1}{4} \partial(v \Psi D \Psi)+\left(\frac{1}{8}-x y\right) D(\Psi \partial \Psi)\right.} \\
& \left.+(x \partial y-y \partial x) \Psi D \Psi+\left(\frac{1}{2} x-y\right) \Psi(D \Psi)^{2}\right] \lambda^{-2} \Lambda .
\end{aligned}
$$

Here $x, y, z, u, v$ are arbitrary functions of $x$ (and $t$ ). Note that $Q_{0}^{-1}$ and the $\bar{\Lambda}^{2}$ term in $Q_{0}^{-2}$ are independent of the arbitrary functions modulo total derivative whereas the other terms are not. The $\bar{\Lambda}^{2}$ belongs to $\widetilde{\mathscr{K}}^{-2}$ and its coefficient

$$
J^{1}=-\frac{1}{4}\left[(D \Psi)^{2}-\Psi \partial \Psi\right]
$$

gives a conservation law.

In the case of $\operatorname{OSp}(2 \mid 2)^{(2)}$ we have $Q_{0}^{0}=0$. It iollows that the right-hand side of Eq. (5.10) vanishes and hence the coefficient $J^{0}$ in $Q_{0}^{-1}$ is also a conservation law. We find that $J^{1}=-\frac{1}{2} D J^{0}$.

D. Hamiltonian $\mathscr{A}$. The operator $\left(M_{k}\right)^{0}$ appearing in the time evolution equation (6.14) is given by

$$
M_{k}=e^{-\mathrm{ad} U}\left(\Lambda^{4 k+2}\right)
$$

and is expanded as

$$
M_{k}=\sum_{i=-\infty}^{4 k+2} A^{i}, \quad A^{i} \in \mathfrak{g}^{i} .
$$

We evaluate $\left(M_{k}\right)^{+}$for $k=1$.

$$
\begin{aligned}
A^{6} & =\Lambda^{6}=4 \lambda^{2} \Lambda^{2}, \\
A^{5} & =-\left[U^{-1}, \Lambda^{6}\right]=4 \lambda^{2} Q^{1}, \\
A^{4} & =-\left[U^{-2}, \Lambda^{6}\right]=4 \lambda^{2} Q^{0}, \\
A^{3} & =-\left[U^{-3}, \Lambda^{6}\right]+\frac{1}{2}\left(\left[U^{-1}\left[U^{-2}, \Lambda^{6}\right]\right]+\left[U^{-2},\left[U^{-1}, \Lambda^{6}\right]\right]\right) \\
& =4 \lambda^{2}\left(-Q_{0}^{-1}-\partial U^{-1}+\left[U^{-1}, Q^{0}\right]+\left[U^{-2}, Q^{1}\right]\right),
\end{aligned}
$$




$$
\begin{aligned}
A^{0}= & -\left[U^{-6}, \Lambda^{6}\right]+\frac{1}{2}\left(\left[U^{-1},\left[U^{-5}, \Lambda^{6}\right]\right]+\left[U^{-5},\left[U^{-1}, \Lambda^{6}\right]\right]\right. \\
& \left.+\left[U^{-2},\left[U^{-4}, \Lambda^{6}\right]\right]+\left[U^{-4},\left[U^{-2}, \Lambda^{6}\right]\right]+\left[U^{-3},\left[U^{-3}, \Lambda^{6}\right]\right]\right) \\
& -\frac{1}{6}\left(\left[U^{-1},\left[U^{-2},\left[U^{-3}, \Lambda^{6}\right]\right]\right]+\cdots\right) \\
= & 4 \lambda^{2}\left\{-\partial U^{-4}+\left[U^{-3}, Q_{0}^{-1}\right]+\left[U^{-2}, Q_{0}^{-2}\right]+\left[U^{-1}, Q_{0}^{-3}\right]\right. \\
& +\frac{1}{2}\left(\left[U^{-3}, \partial U^{-1}\right]+\left[U^{-2}, \partial U^{-2}\right]+\left[U^{-1}, \partial U^{-3}\right]\right) \\
& -\left[U^{-1},\left[U^{-2}, Q_{0}^{-1}\right]\right]-\left[U^{-2},\left[U^{-1}, Q_{0}^{-1}\right]\right] \\
& \left.-\frac{1}{6}\left(\left[U^{-1},\left[U^{-2}, \partial U^{-1}\right]\right]+\left[U^{-2},\left[U^{-1}, \partial U^{-1}\right]\right]\right)\right\} .
\end{aligned}
$$

After substituting the results in Appendix C into Eq. (A.22), we obtain

$$
A^{0}=\left[\partial^{2} D \Psi-2(D \Psi)^{3}+3 \Psi D \Psi \partial \Psi\right] H .
$$

Acknowledgements. The authors would like to thank K. Takasaki and S.-K. Yang for useful conversations. They would also like to thank P. P. Kulish for valuable comments on the manuscript. One of the authors (T. I.) is grateful to M. Scheunert for kindly introducing him to basic notations of Lie superalgebras.

\section{References}

1. Zamolodchikov, A.: Infinite additional symmetries in two-dimensional conformal field theory. Theor. Math. Phys. 65, 1206-1213 (1986)

2. Drinfel'd, V. G., Sokolov, V. V.: Lie algebras and equations of Korteweg-de Vries Types. Sov. J. Math. 30, 1975-2036 (1985)

3. Gervais, J. J., Neveu, A.: Dual string spectrum in Polyakov's quantization (II): Mode separation. Nucl. Phys. B209, 125-145 (1982); Khovanova, T. G.: The Gel'fand-Dikii algebras and the Virasoro algebra. Funct. Anal. Appl. 20, 332-334 (1987); Yamagishi, K.: The KP hierarchy and extended Virasoro algebras. Phys. Lett. B205, 466-470 (1988); Mathieu, P.: Extended classical conformal algebras and the second Hamiltonian structure of Lax equations. Phys. Lett. B208, 101-106 (1988); Bakas, I.: Hamiltonian reduction and conformal symmetries in two dimensions. Phys. Lett. B219, 283-290 (1989); Higher spin fields and the GelfandDickey algebra. Commun. Math. Phys. 123, 627-639 (1989); Fateev, V. A., Lykyanov, S. L.: The models of two-dimensional conformal quantum field theory with $Z_{n}$ symmetry. Int. J. Mod. Phys. A3, 507-520 (1988); Belavin, A. A.: KdV-type equations and W-algebras. Adv. Stud. Pure Math. 19, 117-125 (1989); Smit, J.-D.: A quantum group structure in integrable conformal field theories. Commun. Math. Phys. 128, 1-37 (1990)

4. Eguchi, T., Yang, S.-K: Deformations of conformal field theories and soliton equations. Phys. Lett. B224, 373-378 (1989); Hollowood, T. J., Mansfield, P.: Rational conformal field theories at, and away from, criticality as Toda field theories. Phys. Lett. B226 73-79 (1989); Smirnov, F. A.: The perturbed $c<1$ conformal field theories as reductions of sine-Gordon model. Int. J. Mod. Phys. A4, 4213-4220 (1989); Kupershmidt, B. A., Mathieu, P.: Quantum Kortewg-de Vries like equations and perturbed conformal field theories. Phys. Lett. B227, 245-250 (1989)

5. Mikhailov, A. V., Olshanetsky, M. A., Perelomov, A. M.: Two-dimensional generalized Toda lattice. Commun. Math. Phys. 79, 473-488 (1981)

6. Leites, D. A., Saveliev, M. V., Serganova, V. V.: Embeddings of Lie superalgebra $O S p(1 \mid 2)$ and the associated nonlinear supersymmetric equations. Preprint, IHEP 85-81 (1985); Leznov, A. N., Saveliev, M. V.: Two-dimensional supersymmetric nonlinear equations associated with embeddings of the subsuperalgebra $\operatorname{OSP}(1 \mid 2)$ in Lie superalgebras. Theor. Math. Phys. 61, 1056-1059 (1985)

7. Leznov, A. N., Saveliev, M. V.: Representation of zero curvature for the system of nonlinear partial differential equations $z_{\alpha, z \bar{z}}=\exp (K x)_{\alpha}$ and its integrability. Lett. Math. Phys. 3, 489-494 (1979); Representation theory and integration of nonlinear spherically symmetric 
equations to gauge theories. Commun. Math. Phys. 74, 111-118; (1980); Exactly and completely integrable nonlinear dynamical systems. Acta Appl. Math. 16, 1-74 (1989)

8. Kac, V. G.: Infinite dimensional Lie algebras. Cambridge University Press 1985

9. Olshanetsky, M. A.: Supersymmetric two-dimensional Toda lattice. Commun. Math. Phys. 88, 63-76 (1983): Saveliev, M. V.: Integrable supermanifolds and associated nonlinear equations. Theor. Math. Phys. 59, 560-563 (1984); Integrable graded manifolds and nonlinear equations. Commun. Math. Phys. 95, 199 216: (1984); Andreev, V. A.: Odd bases of Lie superalgebras and integrable equations. Theor. Math. Phys. 72, 758-764 (1988); Ikeda, K.: A supersymmetric extension of the Toda lattice hierarchy. Lett. Math. Phys. 14, 321-328 (1987)

10. Manin, Yu. I., Radul, A. O.: A supersymmetric extension of the Kadomtsev-Petviashvili hierarchy. Commun. Math. Phys. 98, 65-77 (1985)

11. Kac, V. G.: Lie superalgebras. Adv. Math. 26, 8-96 (1977)

12. Frappat, A., Sciarrino, A., Sorba, P.: Structure of basic Lie superalgebras and their affine extensions. Commun. Math. Phys. 121, 457-500 (1989)

13. Zakharov, V. E., Shabat, A. B.: A scheme of integrating nonlinear equation of mathematical physics by the method of inverse scattering problem I and II. Funct. Anal. Appl. 8, 226-335 (1974) and 13, 166-174 (1979)

14. Di Vecchia, P., Ferrara, S.: Classical solutions in two-dimensional supersymmetric field theories. Nucl. Phys. B130, 93-104 (1977); Chaichan, M., Kulish, P. P.: On the method of inverse scattering problem and Bäcklund transformations for supersymmetric equations. Phys. Lett. 78B, 413-416 (1978)

15. Ferrara, S., Girardello, L., Sciuto, S.: An infinite set of conservation laws of the supersymmetric sine-Gordon theory. Phys. Lett. 76B, 303-306 (1978)

16. Mathieu, P.: Superconformal algebra and supersymmetric Korteweg-de Vries equation. Phys. Lett. B203, 287-291 (1988); Supersymmetric extension of the Korteweg-de Vries equation. J. Math. Phys. 29, $2499-2506$ (1988); Bilal, A., Gervais, J.-L.: Superconformal algebra and super-KdV equation. Phys. Lett 211, 95-100 (1988)

17. Kupershmidt, B. A.: A super Korteweg-de Vries equation. Phys. Lett. 102A, 213-215 (1984); Super Korteweg-de Vries equations associated to superextensions of the Virasoro algebra. Phys. Lett. 109A, 417-423 (1985); Gürsus, M., Oğuz, Ö.: A super AKNS scheme. Phys. Lett. 108A, 437-440 (1985); Khovanova, T. G.: Lie superalgebra $O S p(1 \mid 2)$, Neveu-Schwarz superalgebra and superization of Korteweg-de Vries equation. In Group theoretical methods in physics, Proceedings of the Third Yurmala Seminar. Markov, M. A., Man'ko, V. I., Dodonov, V. V. (eds.). The Netherlands: VNU Science Press 1986; Chaichan, M., Kulish, P.P.: Superconformal algebras and their relation to integrable nonlinear systems. Phys. Lett. B183, $169-179$ (1987)

18. Nam, S.-K.: On supersymmetric extended conformal algebras and super KP hierarchy. Int. J. Mod. Phys. A4, 4083-4095 (1989)

19. Kac, V. G.: Infinite-dimensional algebras, Dedekind's $\eta$-functions, classical Möbius function and the very strange formula. Adv. Math. 30, 85-136 (1978)

20. Inami, T., Kanno, H.: $N=2$ super $\mathrm{KdV}$ and super sine-Gordon equations based on Lie superalgebra $A(1,1)^{(1)}$. Yukawa Institute preprint, YITP/K-895

21. Yamanaka, I., Sasaki, R.: Super Virosoro algebra and solvable supersymmetric quantum field theories. Progr. Theor. Phys. 79, 1167-1184 (1988)

22. Date, E., Jimbo, M., Kashiwara, M. Miwa, T.: Transformation groups for soliton equation. In: Proceedings of RIMS symposium on nonlinear integrable systems. Jimbo, M., Miwa, T. (eds). Singapore: World Scientific 1983

23. Mulase, M.: Solvability of the super KP equation and a generalization of the Birkoff decomposition. Invent. Math. 92, 1-46 (1987); Kac, V. G., van de Leur, J. W.: Super boson-fermion correspondence. Ann. Inst. Fourier. Grenoble 37, 99-137 (1987); Ueno, K., Yamada, H.: Super-Kadomtsev-Petviashvili hierarchy and super-Grassmann manifold. Adv. Stud. Pure Math. 16, 373-426(1988); Le Clair, A.: Supersymmetric KP hierarchy: Free field construction. Nucl. Phys. B315, 425-438 (1989)

24. Gel'fand, I. M., Dikii, L. A.: A family of Hamiltonian structures connected with integrable nonlinear differential equations. Preprint No. 136, Inst. Applied Math., Moscow 1978

25. Inami, T., Matsuo, Y., Yamanaka, I.: Extended conformal algebras with $N=1$ supersymmetry. Phys. Lett. B215, 701-705 (1989) 